\title{
Stimulus control in fixed interfood intervals
}

\author{
WILLIAM L. PALYA and MATTHEW T. BOWERS \\ Jacksonville State University, Jacksonville, Alabama
}

\begin{abstract}
The control exerted by various portions of fixed-time and fixed-interval schedules was assessed with a trace-conditioning procedure. The intervals were segmented into 10 bins. In all but 1 of those bins, the stimuli were presented in different random orders on each trial. In $1 \mathrm{bin}$, the stimulus was the same on each trial. The position of this trace stimulus was varied across phases. The results indicated that a trace stimulus can come to control behavior and that differential control can extend to even the second tenth of an interfood interval. The results were interpreted as indicating that traditional explanations of the rate loss in earlier portions of an interfood interval are inadequate and that models such as Palya's (1993) bipolar model or Miller and Schachtman's (1985) comparator model may provide a principled framework with which to understand within-trial effects.
\end{abstract}

Learned behavior is acquired as the result of an individual's experience with the environment. Pavlov (1927) and Thorndike(1898) detailed specific functional relationships that control the overall occurrence of two classes of behavioral adaptations. The commonality in their views was that food presentations function to make the behavior occurring in the local stimulus context more likely on future occurrences of that stimulus situation. The obvious fact that this behavior does not occur constantly nor in every possible circumstance was explained by the plausible notions of stimulus specificity and effort minimization.

This type of argument asserts that reinforcement strengthens some tendency to behave in a context, whereas the absence of reinforcement, coupled with a tendency of the organism to expend the least amount of effort, fails to strengthen behavior or weakens it as the stimulus context becomes more dissimilar. After the fact, this mechanism could account for almost any conceivable outcome with a judicial invocation of strengthening processes, weakening processes, and stimulus-control processes, each of which could possess whatever properties were necessary to account for the obtained effect.

Although it is true that any explanation of behavior must have some way of accounting for increases and decreases in response strength as a function of stimulus differences, any assertion purporting to explain behavioral

The authors gratefully acknowledge the contributions of Donald Walter for data analysis and discussions, Elizabeth Palya for contributions in all phases of this research, and the able assistance of Benjamin Zimmerman. The authors especially thank Sam Cleghorn and Mike Benson, without whose support the research would not have been possible. Contact Donald Walter (walter@ sebac.jsu.edu) for access to raw data files. Additional information pertaining to this research can be viewed on this paper's web appendix at http://sebac.psychology.org/bipolar-trace/. Correspondence and requests for reprints should be addressed to W. L. Palya, Department of Psychology, Jacksonville State University, Jacksonville, AL 36265 (e-mail: palya@ sebac.jsu.edu).

-Accepted by the previous editorial team of Ralph R. Miller. adaptation without some falsifiable systematic rules of application is a tautology. It is the specific predictions of a principled system that define "explanation" (see, e.g., Baum, 1974; Gallistel \& Gibbon, 2000; Killeen, 1994; Rescorla \& Wagner, 1972).

Ferster and Skinner (1957) invoked an informal combination of strengthening, weakening, and stimulus control to explain the increasing rate, or "scallop," across a fixed time (FT) period preceding a reinforced response (a fixed-interval, or FI schedule). They had empirically demonstrated that the onset of the rate increase across an FI was retarded with the addition of a continuously changing explicit stimulus correlated with the passage of time. Following Spence (1956), they concluded that the typical spread of effect to earlier portions of the interval was a failure in stimulus control, because stimuli were not sufficiently discriminable from those in effect at the moment of reinforcement. They went on to postulate that a perfect clock would control "perfect" behavior (i.e., a single response). It is important to note that their stimulus discriminability view was an extrapolated inference rather than a set of functional laws (see, e.g., Baum, 1974; Killeen, 1994).

Their inference has, nevertheless, become enshrined as an explanation of behavior across a fixed interfood interval (see, e.g., Catania, 1998, p. 176; Domjan, 2003, p. 168; Mazur, 1998, p. 155). This occurred in spite of several major omissions. No principled falsifiable specification had been provided: for the spread of strengthening or weakening, for whether the absence of conditioning or inhibitory conditioning was to be expected in the earliest portion of the interval, or for how the net effect of these two processes would be expected to change with changes in procedure.

For example, if an FI scallop is explained by the simple invocation of the animal's inability to clearly discriminate the temporal stimuli, then providing a series of clearly discriminable stimuli across the interval must of necessity control behavior to only the final stimulus. Any 
other result would demonstrate the putative explanatory principle as a tautology or, at best, as incomplete. The invocation of some additional inferred process, such as attention, higher order conditioning, or conditioned reinforcement, would not alter the underlying tautology unless some independent measure of attention, higher order conditioning, or conditioned reinforcement across the interfood interval were available. A number of researchers have shown the inadequacy of simple "loss-of-stimuluscontrol" explanations for the behavior in a fixed interfood interval (Barnet, Cole, \& Miller, 1997; Dinsmoor, Dougan, Pfister, \& Thiels, 1992; Dinsmoor, Lee, \& Brown, 1986; Palya, 1985, 1993; Silva \& Timberlake, 1998).

Other explanatory systems are available for the occurrence of responding in other than the final few moments of a fixed interfood interval. Hull (1952), Dews (1962), and Killeen (1994) have argued that the behavior in the earlier portions of an interfood interval is maintained by reinforcement just as are the final instances of the terminal behavior. These views argue that the behavior in earlier portions of the interval is directly supported by delayed reinforcement, but to a lesser extent than to the final stimulus. Alternatively, Palya (1993) and Palya and Chu (1996) have argued that the second half of an interfood interval would be expected to control higher rates of the terminal behavior, whereas the earlier portion of the first half of the interval would be expected to control higher rates of a behavior other than the terminal behavior. In this view, the behavior across an interfood interval is bipolar.

The intent of the present research was to assess the degree to which lack of stimulus control or lack of attention are adequate explanations for the behavior across fixed interfood intervals. The ultimate goal of the present research program is to explain the same FI scallop described by Ferster and Skinner (1957); the present approach is an attempt to do so by eventually specifying a general description of the occurrence and vector of behavior across an interfood interval and by specifying how behavior would be expected to change with changes in procedure.

The present research was based on a modification of an interfood clock procedure (Palya, 1985). Interfood clocks partition the entire interfood interval into relatively short, discriminable periods. For example, a 60 -sec interfood interval might be partitioned into ten 6 -sec stimulus elements. In a typical interfood clock schedule, the stimuli are presented in a fixed order. A typical control procedure for an interfood clock is a randomized clock during which the stimuli are presented in a different random order on every trial.

In the procedures implemented in the present research, only one stimulus of a 10-element interfood clock had a consistent temporal relationship with the upcoming food presentation. The other nine stimuli were randomly ordered within each trial. Because the fixed stimulus was not contiguous with food presentation and occurred in a fixed position with no other explicit stimulus correlated with reinforcement, this procedure was a trace-conditioning procedure. It differed from a traditional trace procedure in that the remaining portion of the trial (the intertrial interval and the trace interval) was not filled with a single stimulus but, rather, with distinct 6-sec stimuli with a random relationship with the upcoming food presentation. In both the traditional trace and the present trace procedures, only a single noncontiguous stimulus has a predictive relationship with the subsequent reinforcer.

Important aspects of the present procedure were that the fixed stimulus was presented within a context of randomly ordered key colors and the trace stimulus was different for each temporal position tested. These characteristics of the procedure assured that the control by the trace stimulus was an associative effect, rather than the simple result of a very salient disruptive stimulus change occurring at some point in the interfood interval (see, e.g., Farmer \& Schoenfeld, 1966) or the simple increase in the delay to reinforcement of an already established predictive stimulus (Lucas, Deich, \& Wasserman, 1981). An additional benefit of presenting the trace stimulus in a context of random color sequences was that, in most cases, randomized clock stimuli tend to maintain some level of responding throughout all but the earliest portion of an interval (Palya \& Bevins, 1990). This characteristic provides for the detection of either a rate increase or a rate decrease to the trace stimulus (see, e.g., Barnet et al., 1997).

\section{METHOD}

\section{Subjects}

Six experimentally naive adult pigeons obtained from a local supplier were used throughout the experiment. They were housed under continuous illumination in individual cages with free access to water. Each bird received approximately 50 food presentations during each experimental session, or the number just suff icient to maintain the bird at $80 \%$ of its free-feeding weight. Birds requiring supplemental feeding were postfed at least $60 \mathrm{~min}$ after the experimental session. Pelletized laying mash was used for both maintenance feeding and reinforcers.

\section{Apparatus}

Six experimental chambers were used. The interior of each was a $30 \times 30 \times 34 \mathrm{~cm}$ high cube. An unpainted aluminum panel served as one wall of the chamber; the other sides were painted flat white. The stimulus panel had a feeder aperture $5 \mathrm{~cm}$ in diameter, medially located $10 \mathrm{~cm}$ above the grid floor. Three response keys, each $2 \mathrm{~cm}$ in diameter, were located $9 \mathrm{~cm}$ apart, $29 \mathrm{~cm}$ above the grid floor. They required approximately $15 \mathrm{~g}(0.15 \mathrm{~N})$ to operate. Only the center key was used. The translucent Plexiglas key could be transilluminated by a stimulus projector containing color filters. The filters were selected to produce hues that provided roughly equally discriminable intervals plus white (Wright, 1978). They were the following Rosco theatrical gels: red (27), vermilion (25), orange (23), amber (21), yellow (12), green (389), emerald (90), turquoise (95), blue (76), purple (80), and white (62). The key was illuminated throughout all phases of the experiment except during food presentation, when the only illumination was provided by a lamp in the aperture above the food magazine. Two houselights, directed upward, were located on the stimulus panel $32 \mathrm{~cm}$ above the grid floor. Ventilation was provided by an exhaust fan mounted on the outside of the chamber. A white-noise generator provided ambient masking noise within the chamber. 
Stimulus events were controlled and keypecks were recorded by a computer network consisting of a host computer and a separate control computer for each chamber (Palya \& Walter, 1993). The host computer archived the time of each stimulus and response event in 1-msec ticks. Subsequent data extraction and analysis routines provided the resulting behavioral indices. Complete raw-data event logs of all research are maintained for at least 10 years and are available for electronic download upon request (see Acknowledgment).

\section{Procedure}

In general, consecutive phases of the experiment assessed the ability of an explicit stimulus in various specific temporal positions within an interfood interval to control a behavior change. The procedure used a single fixed stimulus embedded in a context of randomly ordered stimuli to minimize the alternate explanations that would be available if a single highly salient stimulus had been presented in an otherwise unchanging context. In order to assess the generality of the effect, two different stimulus sequences were used, and both a response-independent and a response-dependent food presentation procedure were implemented. All procedures were based on a 10 -stimulus, 60 -sec interfood clock and were implemented on the center key. Table 1 documents the procedure for each bird for each of the 24 phases of the experiment. It also indicates the length of exposure to each phase.

All the pigeons were initially magazine-trained to a criterion of approaching and eating from the food magazine within $3 \mathrm{sec}$ on three consecutive presentations. During magazine training, the keys were dark. Each subsequent phase continued until each bird's behavior reached steady state (no consistent session-to-session trends over the last five sessions) as determined by visual inspection of daily response rates to each bin plotted as a function of session number for each bird, sufficient data were collected for potential analyses, and until the procedural change could be implemented within the day-to-day lab activities. Typically, an informal lab policy of postponing any change in procedure until the next fivesession boundary was used whenever it was convenient. The intent of the latter criterion was to reduce the likelihood that some atypical local aspect of the behavior would be consistently included in the data representing the effects of a phase.

Response-independent procedures. As is documented in Table 1, the first experimental treatment (Phase 1) exposed the birds to a 10-position, 60-sec interfood clock as a baseline. Two different color sequences were used. The first group (Birds 456, 516, and 552) received red, vermilion, orange, amber, yellow, green, emerald, turquoise, blue, and purple followed by $3 \mathrm{sec}$ of food presentation, irrespective of behavior; the second group (Birds 606, 609, and 622) received orange, green, turquoise, emerald, blue, purple, yellow, red, amber, and vermilion followed by food presentation, irrespective of behavior. Phase 1 was in effect for 71 sessions.

The second phase, also implemented as a baseline, was a traditional single-stimulus trace-conditioning procedure. The birds in Group 1 received a 48-sec ITI with a white keylight followed by $6 \mathrm{sec}$ of a blue keylight followed by $6 \mathrm{sec}$ of a white keylight. The ITI was also white for Group 2, but the trace stimulus was amber for the 3 birds of that group. This simple trace procedure was in effect for 55 sessions.

In Phase 3, the intertrial interval and the trace interval were changed to a random sequence (without replacement) of the remaining nine clock stimuli on each trial. As a result, any color but that of the trace stimulus could occur in any of the remaining nine positions of the clock on a given trial (designated as $\times$ in the table). Position 9 was always blue for Group 1 and always amber for Group 2. The birds were exposed to this procedure for 42 sessions.

In the next seven phases, the trace stimulus was changed to successively earlier clock positions in order to determine the degree to which a trace stimulus in the various positions of an interfood in- terval could come to control a behavior change. As can be seen in Table 1, the trace stimulus for a specif ic bin was the hue correlated with that temporal position in the first phase and was, therefore, a different hue in each phase. Each of these phases was in effect for approximately 40 sessions, with the exception of Phase 4 , which was in effect for 100 sessions.

Response-dependent procedures. Phase 11 was a baseline for the subsequent phases, all of which had a response requirement. The procedure randomized all 10 stimuli and required a response following the elapse of $60 \mathrm{sec}$ since the preceding food presentation (6 sec after the onset of the last stimulus). The final stimulus remained in effect until the reinforced response occurred. Phase 11 was continued for 30 sessions. The response requirement was added for two reasons. First, it was expected to increase the response rates of the birds with marginal rates, and, second, it would demonstrate the rate changes to the trace stimuli with a somewhat different procedure.

In the next nine phases, the ability of trace stimuli in the first half of the interfood interval to control behavior was assessed. The trace stimulus was moved to later bins $(2,3,4,5$, and 6$)$, then to earlier bins $(5,4$, and 3$)$ and, finally, as a local baseline, to Bin 6 . These phases were implemented to provide multiple assessments of the portion of the interfood interval of most interest. Because Bin 6 was the first bin past the midpoint of the interfood interval, the behavior to a fixed stimulus in that position was used as the local baseline. Phase 21 was a return to the fully randomized baseline procedure of Phase 11 in order to reestablish an exemplar of the behavior under a totally randomized FI clock.

Control procedures. The final three phases were implemented to evaluate potential explanations for the obtained rate decreases to the trace stimuli. Phase 22 was in effect for 30 sessions. This procedure provided information about the effect of simple nonreinforcement on an otherwise randomized clock stimulus. This phase implemented a randomly positioned trace stimulus that was never paired with food or even with the stimulus that preceded food. Any of the 10 stimuli, including the stimulus appropriate for Bin 6, could occur in the first eight positions. The table designates the possibility of either a nontrace random stimulus (i.e., $X$ ) or the trace stimulus itself (e.g., G or P) as $\times \mathrm{G}$ or $\times \mathrm{P}$. Any of the 10 stimuli except the stimulus appropriate for Bin 6 could occur in either of the final two bins. As in earlier phases, this is designated as $X$ in the table. This procedure was considered a very strong, explicitly unpaired procedure (Rescorla, 1967).

Phase 23 was in effect for 45 sessions and implemented what was essentially the converse control procedure. The trace stimulus was consistently presented in Bin 6, but was not excluded from occurring in the final bin. As a result, the trace stimulus also occurred in the final position on $10 \%$ of the trials (as before, if either a randomized nontrace stimulus or the trace stimulus itself occurred in a bin, it is designated $\times \mathrm{G}$ or $\times \mathrm{P}$ in the table). The results from Phase 23 provided information on the control exerted by a stimulus presented in a fixed temporal position early in the interval, when that stimulus was also paired with primary reinforcement. This procedure removed any detrimental effect of the stimulus's simply being unpaired with reinforcement. The final phase (Phase 24) was in effect for 45 sessions and was a return to the basic procedure with a fixed trace stimulus in Bin 6. Its conditions were identical to those of Phases 16 and 20. Phase 24 provided a local comparison for the preceding control procedures.

\section{RESULTS}

In the present study, we examined the ability of a trace stimulus to control a positive or negative behavior change when that trace stimulus was in various specific temporal positions of an interfood interval. The control exerted 
Table 1

Elements of Procedures Used in the Experiment

\begin{tabular}{|c|c|c|c|c|c|c|c|c|c|c|c|c|c|c|c|c|c|c|c|c|c|c|}
\hline \multirow[b]{2}{*}{ Phase } & \multirow{2}{*}{$\begin{array}{c}\text { Number of } \\
\text { Sessions } \\
\end{array}$} & \multirow[b]{2}{*}{$\mathrm{R}$ ? } & \multicolumn{10}{|c|}{ Bin } & \multicolumn{10}{|c|}{ Bin } \\
\hline & & & 1 & 2 & 3 & 4 & 5 & 6 & 7 & 8 & 9 & 10 & 1 & 2 & 3 & 4 & 5 & 6 & 7 & 8 & 9 & 10 \\
\hline & & & \multicolumn{10}{|c|}{$\begin{array}{c}\text { Group } 1 \\
\text { Birds } 456,516,552\end{array}$} & \multicolumn{10}{|c|}{$\begin{array}{c}\text { Group } 2 \\
\text { irds } 606,609,622\end{array}$} \\
\hline 1 & 71 & & $\mathbf{R}$ & V & O & $\mathbf{A}$ & $\mathbf{Y}$ & $\mathbf{G}$ & $\mathbf{E}$ & $\mathbf{T}$ & B & $\mathbf{P}$ & O & $\mathbf{G}$ & $\mathbf{T}$ & $\mathbf{E}$ & B & $\mathbf{P}$ & $\mathbf{Y}$ & $\mathbf{R}$ & $\mathbf{A}$ & $\mathbf{V}$ \\
\hline 2 & 55 & & W & W & W & W & W & W & W & W & B & W & W & $\mathrm{W}$ & W & $\mathrm{W}$ & $\mathrm{W}$ & W & $\mathrm{W}$ & $\mathrm{W}$ & $\mathbf{A}$ & W \\
\hline 3 & 42 & & $\times$ & $x$ & $\times$ & $\times$ & $\times$ & $\times$ & $\times$ & $\times$ & B & $x$ & $\times$ & $x$ & $x$ & $\times$ & $\times$ & $x$ & $x$ & $\times$ & $\mathbf{A}$ & $\times$ \\
\hline 4 & 100 & & $\times$ & $\times$ & $\times$ & $\times$ & $\times$ & $\times$ & $\times$ & $\mathbf{T}$ & $\times$ & $x$ & $\times$ & $\times$ & $\times$ & $\times$ & $\times$ & $\times$ & $\times$ & $\mathbf{R}$ & $\times$ & $\times$ \\
\hline 5 & 45 & & $\times$ & $x$ & $x$ & $x$ & $\times$ & $x$ & $\mathbf{E}$ & $x$ & $\times$ & $x$ & $x$ & $x$ & $x$ & $x$ & $x$ & $x$ & $\mathbf{Y}$ & $\times$ & $\times$ & $\times$ \\
\hline 6 & 35 & & $x$ & $\times$ & $\times$ & $x$ & $x$ & $\mathbf{G}$ & $x$ & $\times$ & $\times$ & $x$ & $x$ & $x$ & $\times$ & $x$ & $x$ & $\mathbf{P}$ & $\times$ & $\times$ & $\times$ & $x$ \\
\hline 7 & 40 & & $x$ & $x$ & $\times$ & $x$ & $\mathbf{Y}$ & $x$ & $x$ & $\times$ & $x$ & $x$ & $x$ & $\times$ & $\times$ & $x$ & B & $x$ & $\times$ & $x$ & $\times$ & $x$ \\
\hline 8 & 40 & & $x$ & $\times$ & $\times$ & $\mathbf{A}$ & $\times$ & $\times$ & $\times$ & $x$ & $\times$ & $x$ & $\times$ & $\times$ & $\times$ & $\mathbf{E}$ & $x$ & $x$ & $\times$ & $\times$ & $\times$ & $\times$ \\
\hline 9 & 45 & & $x$ & $x$ & O & $x$ & $x$ & $x$ & $\times$ & $\times$ & $\times$ & $x$ & $x$ & $x$ & $\mathbf{T}$ & $x$ & $x$ & $x$ & $\times$ & $\times$ & $\times$ & $x$ \\
\hline 10 & 52 & & $\times$ & V & $\times$ & $\times$ & $\times$ & $\times$ & $\times$ & $x$ & $\times$ & $\times$ & $x$ & $\mathbf{G}$ & $\times$ & $\times$ & $x$ & $\times$ & $\times$ & $\times$ & $\times$ & $\times$ \\
\hline 11 & 30 & $*$ & $\times$ & $x$ & $\times$ & $\times$ & $x$ & $x$ & $\times$ & $x$ & $x$ & $x$ & $x$ & $x$ & $x$ & $x$ & $x$ & $x$ & $x$ & $\times$ & $\times$ & $x$ \\
\hline 12 & 35 & $*$ & $\times$ & $\mathbf{V}$ & $x$ & $x$ & $\times$ & $x$ & $\times$ & $\times$ & $\times$ & $x$ & $\times$ & $\mathbf{G}$ & $\times$ & $\times$ & $\times$ & $\times$ & $\times$ & $\times$ & $\times$ & $\times$ \\
\hline 13 & 40 & $*$ & $x$ & $\times$ & O & $x$ & $x$ & $x$ & $\times$ & $x$ & $\times$ & $x$ & $\times$ & $x$ & $\mathbf{T}$ & $x$ & $x$ & $x$ & $\times$ & $\times$ & $\times$ & $x$ \\
\hline 14 & 60 & $*$ & $\times$ & $\times$ & $\times$ & $\mathbf{A}$ & $\times$ & $\times$ & $\times$ & $x$ & $\times$ & $\times$ & $\times$ & $\times$ & $\times$ & $\mathbf{E}$ & $\times$ & $\times$ & $\times$ & $\times$ & $\times$ & $\times$ \\
\hline 15 & 45 & $*$ & $x$ & $\times$ & $x$ & $x$ & $\mathbf{Y}$ & $x$ & $\times$ & $\times$ & $\times$ & $x$ & $x$ & $\times$ & $\times$ & $x$ & B & $x$ & $\times$ & $\times$ & $\times$ & $\times$ \\
\hline 16 & 35 & $*$ & $\times$ & $x$ & $x$ & $x$ & $\times$ & $\mathbf{G}$ & $\times$ & $\times$ & $\times$ & $x$ & $x$ & $x$ & $\times$ & $\times$ & $\times$ & $\mathbf{P}$ & $\times$ & $\times$ & $\times$ & $\times$ \\
\hline 17 & 45 & $*$ & $\times$ & $\times$ & $\times$ & $x$ & $\mathbf{Y}$ & $x$ & $\times$ & $\times$ & $\times$ & $\times$ & $\times$ & $\times$ & $x$ & $\times$ & $\mathbf{B}$ & $x$ & $\times$ & $\times$ & $\times$ & $\times$ \\
\hline 18 & 45 & $*$ & $x$ & $\times$ & $x$ & $\mathbf{A}$ & $\times$ & $x$ & $\times$ & $\times$ & $\times$ & $x$ & $x$ & $x$ & $\times$ & $\mathbf{E}$ & $x$ & $x$ & $\times$ & $\times$ & $x$ & $\times$ \\
\hline 19 & 40 & $*$ & $x$ & $x$ & $\mathbf{O}$ & $x$ & $\times$ & $x$ & $\times$ & $\times$ & $\times$ & $x$ & $x$ & $x$ & $\mathbf{T}$ & $\times$ & $x$ & $x$ & $x$ & $\times$ & $\times$ & $\times$ \\
\hline 20 & 40 & $*$ & $\times$ & $x$ & $x$ & $x$ & $\times$ & $\mathbf{G}$ & $\times$ & $\times$ & $x$ & $x$ & $x$ & $x$ & $\times$ & $x$ & $x$ & $\mathbf{P}$ & $x$ & $\times$ & $\times$ & $\times$ \\
\hline 21 & 45 & $*$ & $\times$ & $\times$ & $\times$ & $\times$ & $\times$ & $\times$ & $\times$ & $\times$ & $\times$ & $\times$ & $x$ & $\times$ & $\times$ & $\times$ & $\times$ & $\times$ & $\times$ & $\times$ & $\times$ & $\times$ \\
\hline 22 & 30 & $*$ & $\times \mathbf{G}$ & $\times \mathbf{G}$ & $\times \mathbf{G}$ & $\times \mathbf{G}$ & $\times \mathbf{G}$ & $\times \mathbf{G}$ & $\times \mathbf{G}$ & $\times \mathbf{G}$ & $\times$ & $x$ & $\times \mathrm{P}$ & $\times \mathrm{P}$ & $\times \mathrm{P}$ & $\times \mathrm{P}$ & $\times \mathrm{P}$ & $\times \mathrm{P}$ & $\times \mathrm{P}$ & $\times \mathrm{P}$ & $\times$ & $x$ \\
\hline 23 & 45 & $*$ & $\times$ & $x$ & $\times$ & $x$ & $\times$ & $\mathbf{G}$ & $\times$ & $\times$ & $\times$ & $\times \mathbf{G}$ & $\times$ & $\times$ & $\times$ & $\times$ & $\times$ & $\mathbf{P}$ & $\times$ & $\times$ & $\times$ & $\times \mathrm{P}$ \\
\hline 24 & 45 & $*$ & $x$ & $x$ & $x$ & $x$ & $x$ & $\mathbf{G}$ & $x$ & $x$ & $\times$ & $x$ & $x$ & $\times$ & $x$ & $x$ & $\times$ & $\mathbf{P}$ & $x$ & $x$ & $\times$ & $\times$ \\
\hline
\end{tabular}

Note- $\mathrm{R}$ ? = Response Requirement? An asterisk in the $\mathrm{R}$ ? column denotes that there was a response requirement for the indicated phase. Letters in boldface indicate key colors that occurred in the same position in every trial of the corresponding phase, and are the colors detailed in the Procedure section $(\mathrm{R}=$ red, $\mathrm{V}=$ vermilion, etc.). $\mathrm{W}$ indicates a white keylight, and $\times$ represents the random selection (without replacement) of any color except that of the trace stimulus. The notations $\times \mathrm{G}$ and $\times \mathrm{P}$ indicate that any key color, including the trace stimulus (green and purple), could be presented.

by each trace stimulus was assessed by noting changes in the rate of pecking to that stimulus as compared with the rate controlled by the randomized stimuli in the adjacent bins. The measure was, therefore, a within-trial, withinbird index capable of detecting either a rate increase or a rate decrease controlled by the trace stimulus. This strategy was feasible because, for most birds, response rates systematically increase across consecutive bins of a fixed interfood interval with randomized clock stimuli and very rarely show a reversal in rate to a single bin (Palya \& Bevins, 1990).

\section{Dependent Variables}

A simple criterion for accepting that behavior had come under the control of the trace stimulus was a rate to the trace stimulus that was either higher or lower than that to both of the adjacent bins. In such cases, the behavior to the trace stimulus was clearly different from the trend across the interfood interval. The low power of this relatively stringent criterion would lead to error on the side of rejecting the evidence for control by a trace stimulus when in fact it had occurred. This was adequate for the purposes of the present research, in that even with this stringent criterion, there was evidence that a behavior change could be acquired to the longest trace interval tested. An arrow above a bin in Figures 1, 2, and 3 des- ignate that the rate was either higher (point up) or lower (point down) than the rates to both of the adjacent stimuli for that bin for that bird.

A second index of the control exerted by the trace stimuli was also used. It was the deviation of the trace stimulus response rate from a rate predicted by a spline. A spline is a standard mathematical procedure which determines a best fit curved line (cubic polynomial) through data points (Press, Flannery, Teukolsky, \& Vetterling, 1989). This more powerful index allowed a detection of control that was less extreme than did the stringent criterion. For the present analysis, the interest was in providing for the positive acceleration that characterizes behavior in a fixed interfood interval without simply recreating the idiosyncrasies of the original distribution, so the lowest curvature function that would pass through all but the data point for the predicted bin was used. A spline's advantage over a simple linear predictor lies in the greater fidelity of its curve to the acceleration in response rate across the interfood interval.

The frames presented in Figures 1, 2, and 3 provide the response rate to each bin across the interfood interval for each bird under each procedure. The data for the 6 birds are presented individually in the six columns of each figure. There did not appear to be sufficient evidence to consider the effects of the two hue orders as dif- 
ferent; therefore, the groups are not discussed separately. Consecutive phases are presented in successively lower frames. The response-independent procedures (Phases 1 through 10) are presented in Figure 1, and the responsedependent procedures (Phases 11 through 21) are presented in Figure 2. The control procedures (Phases 22 through 24 ) are depicted in Figure 3. The 10 bars in each frame provide the mean response rate across the last five sessions for the consecutive bins in the interfood interval for the indicated bird in the indicated phase. Markers below the $x$-axis in a frame designate the bin that had the trace stimulus (the same key color on every trial).

\section{Specific Effects}

Baselines. The upper row of frames in Figure 1 (a clocked FT schedule) and the top and bottom row of frames in Figure 2 (randomly clocked FI schedules) provide baseline performances against which the effects of the various trace stimulus procedures can be compared. Most often, but not necessarily, the rate increased in each consecutive bin across the interfood interval. Bird 456 was a clear exception, with 9 of 27 bin-to-bin transitions in the three baseline phases showing a decrease. Additionally, Bird 609 in Phase 1 and Bird 552 in Phase 11 showed an instance of a bin-to-bin decrease in rate.

FT versus FI. As can be seen in a comparison between Figure 1 and Figure 2, there appeared to be more responding, and a greater tendency for responding to start earlier and to increase across the final portion of the interval, when the response dependency was in effect. Because the intent of adding the response requirement had been to enable a better assessment of the behavior controlled by trace stimuli in the first half of the interfood interval, the behavior change served the purposes of the experiment. However, even though it is generally accepted that response dependencies increase the response rate over FT schedules, the present procedure was not sufficient to establish that as a causal effect.

Trace. The effect of primary interest in the present study was the ability of the trace stimulus to come to control a behavior change. An overall impression of the general effectiveness of the trace stimulus in controlling a behavior change can be obtained by scanning Figures 1 and 2 and noting a rate change when a trace stimulus was in a particular bin, and the absence of a rate change in either the adjacent bins or in the same bin when the trace stimulus was absent. The behavior change to the trace stimulus exceeded the stringent criterion in 33 of the 48 treatment frames in Figure 1, and in 30 of the 54 treatment frames in Figure 2. The effect of position on the responding controlled by the trace stimulus within each bird can be seen by viewing the columns in Figures 1 and 2 .

Figure 1 shows the obtained behavior under the response-independent procedure when the trace stimulus was in Bins 9, 8, 7, 6, 5, 4, 3, and 2, successively. The data for the successive phases are provided in successively lower rows. There was an increase in the rate of response to the trace stimulus when it was in the final portion of the interfood interval (Bin 9, and also in Bin 8 for Birds 606 and 609). A rate decrease typically occurred to the trace stimulus when the fixed stimulus was in the remaining positions. In the case of Bird 456, the effect of the trace stimulus was clearly apparent in every position in spite of the fact that this bird had the most irregular distribution across the interfood interval. On the other hand, a scan of the column for Bird 516 shows that this bird rarely showed a rate change that exceeded the stringent criterion. The relatively low response rates of this bird undercut the ability of the present procedure to reveal rate changes attributable to the trace stimulus in this bird.

The same general effect seen in Figure 1 was revealed when rate change to the trace stimulus was indexed as a deviation from a spline (or from a linear increase in rate). Initially, there was a higher rate than that predicted by the spline (when the trace was in Bin 9 or in Bins 8 and 9). Subsequently, when the trace stimulus was positioned earlier and earlier in the interval, every bird showed lower rates than those predicted by the spline until Bin 2 or 3, when the deviation dropped to zero for Birds 606, 622 , and 552 .

Figure 2 illustrates the effect of the trace stimulus in the early portion of the interval when the overall response rate was increased with the addition of a response requirement. In general, a pattern similar to that obtained under the FT schedule occurred. There was a decrease in rate to the trace stimulus in some birds even in the earliest portions of the interval, and, again, this effect did not necessarily occur in every bird under every procedure. Overall, the stringent criterion was met in slightly more than half the cases, even though only the initial portions of the interfood interval were tested (Bins 2 through 6). This was a strong effect, in that trace conditioning is not typically expected to occur at all to stimuli in the first half of an interfood interval (Gibbon \& Balsam, 1981), and response rates in the early portion of the interval remained low, making it difficult to demonstrate large rate changes. The general similarity in the effects of the trace stimulus under the FI procedure with those under the FT schedule was also evident when the effect of the trace stimulus was indexed as a deviation from a spline (or a linear increase). The behavior of Bird 456 failed to reverse following the placement of a trace stimulus in Bin 2 for the second time. Subsequently, this bird exhibited a rate decrease in Bin 2 even under baseline conditions. As a result, for this bird, the lower rate to the trace in Bin 2 cannot be interpreted as an effect of the trace stimulus.

Control. In Figure 3, the results of the control procedures implemented to evaluate potential explanations for the obtained suppression to the trace stimuli are presented. The top row depicts the data from the randomly positioned but explicitly unpaired stimulus. This procedure provided information about the response rate maintained by a stimulus when it was explicitly unpaired with the stimulus preceding food presentation, as well as being explicitly unpaired with food presentation itself. The bars depict the rate to each bin when one of the nine 

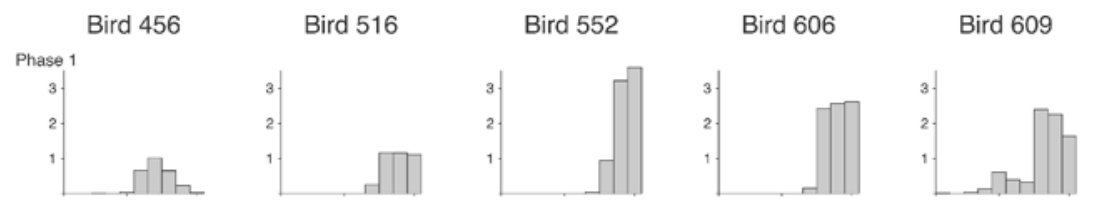

Bird 622
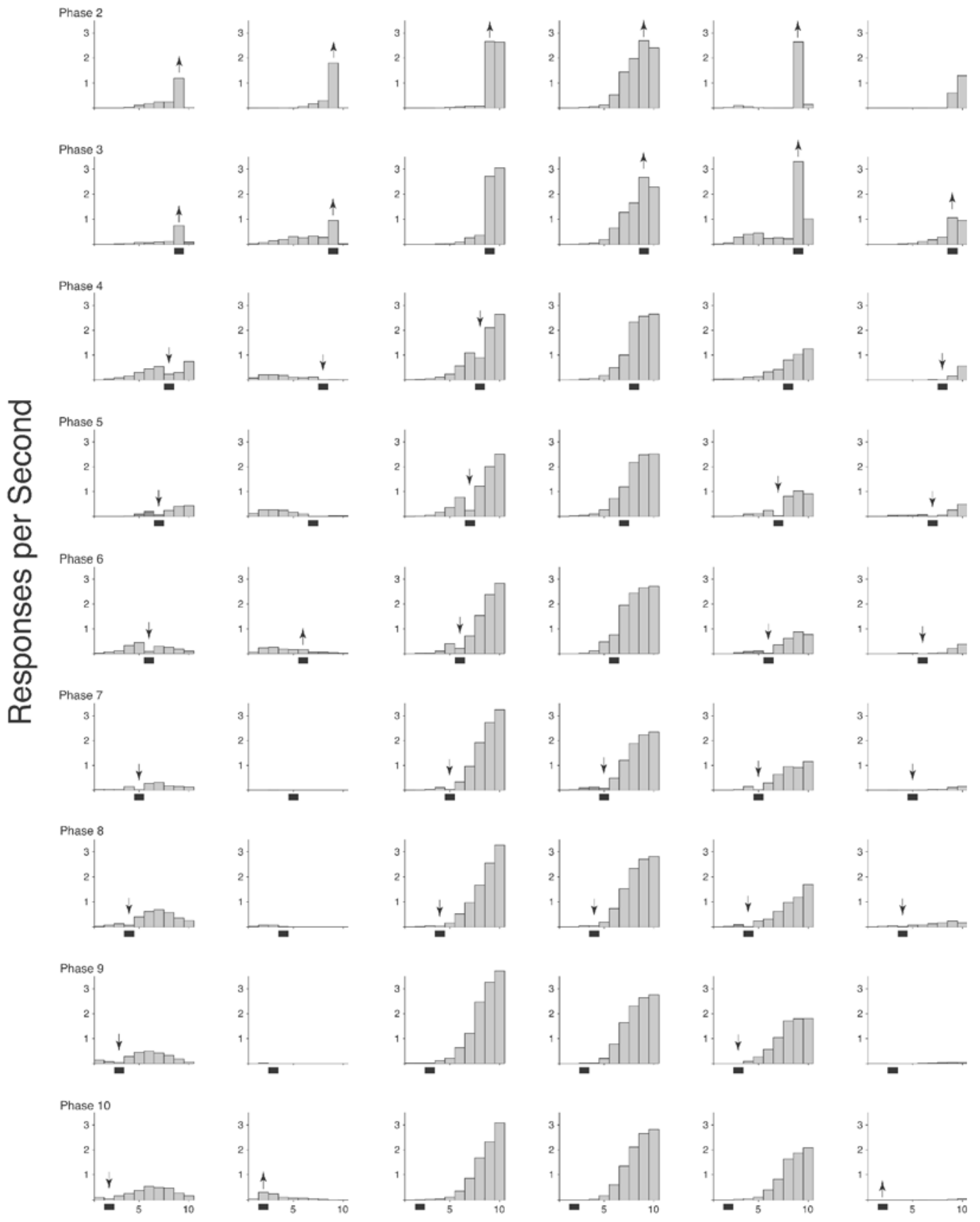

Bin

Figure 1. The response rate to each bin across the interfood interval for each bird under each procedure in Phases 1 through 10. The data for the 6 birds are presented individually in the six columns. Consecutive phases are presented in successively lower frames. The bars in each frame depict the mean response rate across the last five sessions for each consecutive bin in the interfood interval for the indicated bird in the indicated phase. Markers below the $x$-axis in a frame designate the bin that had the same key color on every trial (the trace stimulus). An arrow above that bin designates that the rate was either higher (point up) or lower (point down) than the rates to both of the adjacent stimuli for that bin for that bird. 

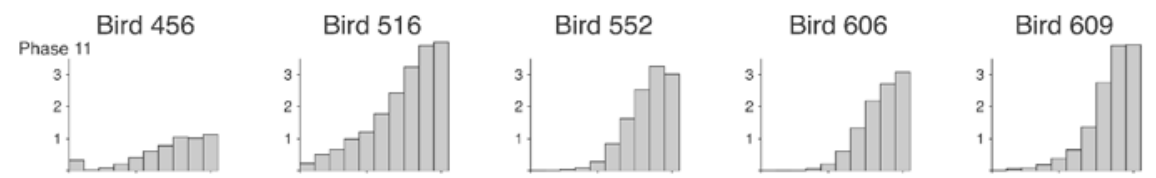

Bird 622
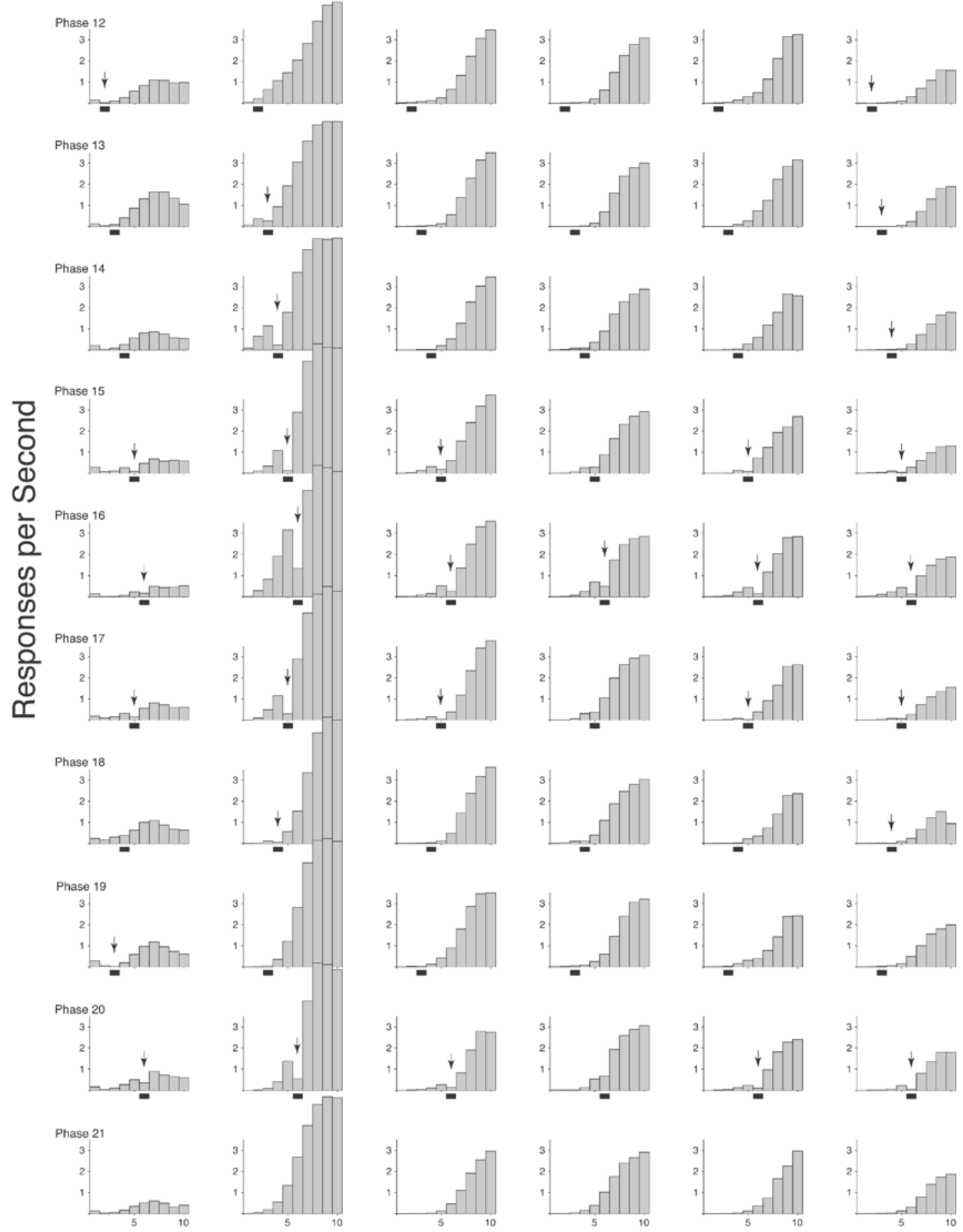

Bin

Figure 2. The response rate to each bin across the interfood interval for each bird under each procedure in Phases 11 through 21. See Figure 1 for a detailed explanation. 


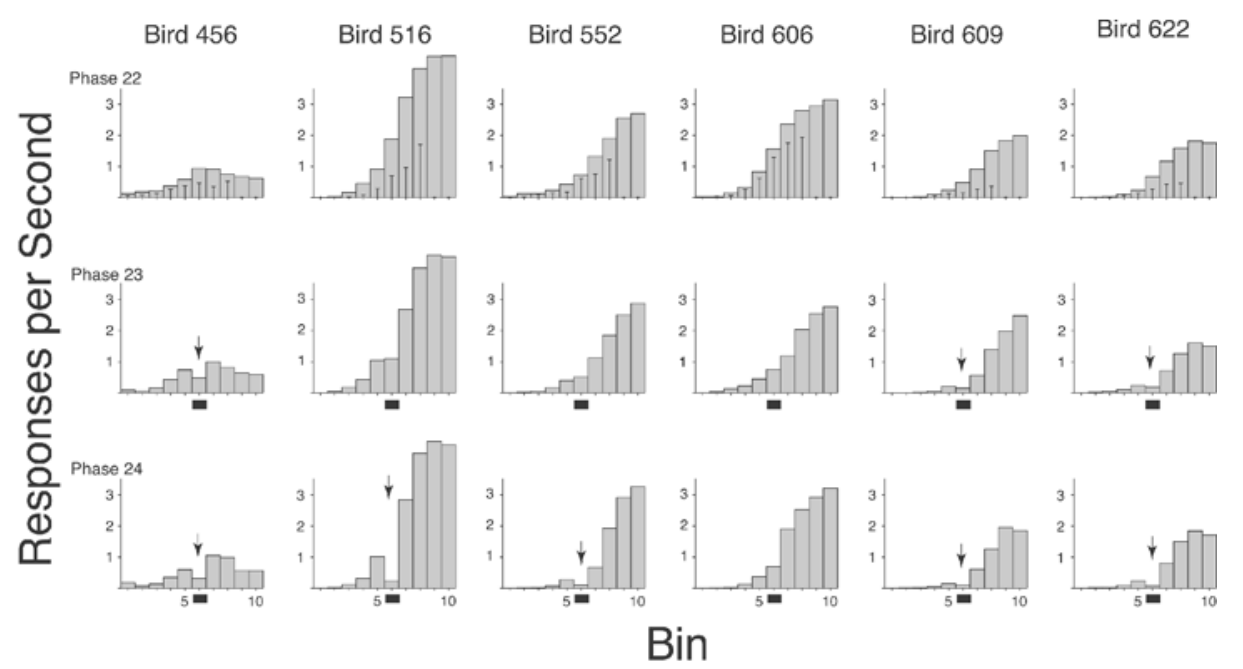

Figure 3. The response rate to each bin across the interfood interval for each bird under each procedure in Phases 22 through 24 . The bars in each frame in the top row designate the rate to random stimuli in each bin, whereas the whiskers indicate the rate in that bin when the unpaired stimulus was presented in that position. See Figure 1 for a detailed explanation.

randomly positioned, paired stimuli occurred in that bin, whereas the whiskers indicate the rate in the bin when the randomly positioned but explicitly unpaired stimulus was presented in that position. There are no whiskers in the last two bins of each frame, because the randomly positioned but unpaired stimulus never occurred in those positions. As can be seen, the response rate in a bin was lower when the explicitly unpaired stimulus occurred in that bin.

The middle row of frames depicts the results from the trace-plus-pairing, or converse, procedure. This procedure provided information on the rate that would be maintained by a fixed trace stimulus when it was not explicitly unpaired. Even though the trace stimulus was occasionally paired with food presentation and therefore provided an imperfect predictor of nonreinforcement, the behavior to the trace stimulus met the stringent criterion for suppression of responding in 3 of the 6 birds. Every bird showed some degree of suppression in that all birds had a negative trace-bin-to-spline deviation. Any rate loss to the fixed stimulus under this procedure could not be attributed to its being explicitly unpaired.

The bottom row of frames in Figure 3 illustrates the results obtained when the basic trace procedure used throughout Experiments 1 and 2 (fixed stimulus in Position 6) was reinstituted. These data provide for a local comparison between the control procedures and a standard trace procedure. The baseline trace procedure generated suppression, which exceeded the stringent criterion in 5 of the 6 birds. Every bird had a lower rate to the trace than that predicted by the spline.

The data from the control procedures presented in Figure 3 indicate that it is possible to implement sufficient separation between a randomly presented stimulus and food presentation to suppress responding to that stimulus. However, separation from food presentation is not a necessary condition for suppression. Responding is suppressed to trace stimuli early in the interfood interval even when those stimuli are also paired with food presentation.

Reliability. The difference between the obtained rate and that predicted by the spline was used as a basis for determining the reliability of the suppression in response rate to the trace stimulus across procedures. The distribution of trace-bin-to-prediction deviations was compared to the distribution of nontrace-bins-to-prediction deviations in the treatment phases. The reliability of the difference between the two distributions was assessed with a Tukey-Kramer HSD test. The probability that the two distributions would differ as much or more by chance was less than .05 for each of the 6 birds.

\section{Summary}

Frequency. As a simple graphical summary of the effectiveness of the procedures across birds and replications, the number of birds whose rates were either above or below the rates in both of the adjacent bins are presented in Figure 4. The 10 consecutive bins are provided across the $x$-axis, whereas the $y$-axis indicates the mean number of birds with rates above (above $x$-axis) or below (below $x$-axis) those in the adjacent bins. As a result, the dependent measure could vary from +6 to -6 . The data provided for each bin is based on all phases in which that bin was tested. For example, the treatment effect for Bin 9 is based on the data from Phase 3, whereas the treatment effect for Bin 6 is based on the data from Phases 6 , 16,20 , and 24 . The baseline frame provides the data for Bins 2 through 9 for every bird during Phases 1, 11, and 21. 


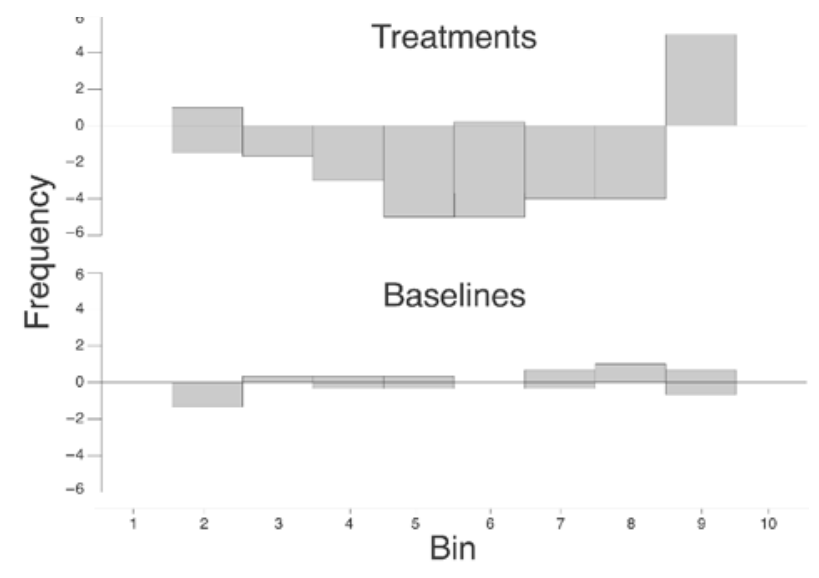

Figure 4. The mean number of birds whose rates exceeded the stringent criterion in each bin under the treatment (upper frame) and baseline (lower frame) conditions. Birds with rates above the adjacent bins are depicted above the axis. Birds with rates below the adjacent bins are depicted below the axis.

The top frame in Figure 4 shows that a monotonic increase in rate was not typical of birds when a trace stimulus occurred. Most typically birds had a response rate that exceeded those of the adjacent bins if the trace stimulus occurred in Bin 9, and a response rate that was below those of the adjacent bins when the trace stimulus was presented in Bins 3 through 8 . The bottom frame of Figure 4 shows that during baseline, few birds had a rate to a bin that exceeded or fell below those of both of the adjacent bins. This would be expected, given that the most typical pattern across the interval was a steady increase in rate.

Trace position. As a graphical summary of the effectiveness of the trace procedure as a function of position in the interfood interval, the deviation in responding controlled by the trace stimuli is presented in Figure 5. The presentation format summarizes both the relative magnitude of the increase or decrease in the behavior and the absolute rate of the adjacent stimuli for the 6 individual birds across 17 trace procedures as well as 3 baseline procedures.

The data in Figure 5 are based on the mean response rates across the last five sessions of each phase. The left column presents the responding to the trace stimuli, and the right column presents the responding to the same bins under the baseline procedures. The six rows of the figure present the data for the 6 birds individually. The data for each of the tested bins ( 2 through 9 ) across the interfood interval are depicted by data points above or below that position along the $x$-axis. Multiple data points in a bin indicate multiple determinations for that bin. The extent of the deviation above or below the zero point on the $y$-axis in each frame indicates the relative degree of deviation above or below the rate predicted by a spline.

The absolute rate from which the relative deviation was indexed - the second critical aspect of these datais presented as an independent dimension. Whereas the distance from the $x$-axis designates the percentage rate change, the diameter of the data point indicates the mean response rate to the adjacent bins. In this way, the visual weight of the data point representing a given percentage change indicates the absolute response rate of its context (Tufte, 2001). For example, a large dot well below the zero point would indicate that the designated trace stimulus controlled a substantial rate decrease in both relative and absolute terms (such as a response rate of one peck per second in the trace stimulus with an average of four pecks per second in the adjacent bins). A well-separated small dot above the axis would indicate that the trace stimulus controlled a large relative increase in rate, but that the absolute rate in that portion of the interval had been low (such as a total of eight pecks in the trace bin in the context of a total of only two pecks in the two adjacent bins).

As can be seen in a comparison of the treatment and baseline columns with respect to percent change (distance from $x$-axis), direction of change (positive or negative $y$ value), and base rate (dot diameter), it is apparent that the magnitude of the treatment effect (left column) generally exceeded the magnitude of similar comparisons in the baseline (right column) in all but the earliest portion of the interval. Although the deviations in the baseline tend toward zero throughout most of the interfood interval with an inconsistent effect in the very earliest portion, the trace stimulus controlled a higher rate in the final portions of the interval and a lower rate in the earlier portions of the interval. It also showed an inconsistent effect in the earliest portion of the interval. The inconsistent effects in the earliest portions of the interval are not entirely surprising, in that the response rates were very low in the very earliest portions of the interval.

\section{DISCUSSION}

Figure 4, which summarized the frequency across birds of exceeding the stringent criterion, and Figure 5, which summarized the magnitude of the effect for each bird in each bin, provided two different overviews of the reliable behavior change controlled by trace stimuli throughout the interfood interval as were detailed in Figures 1, 2, and 3. That behavior change demonstrates that it can no longer be asserted, without support, that behavior in the interfood interval is the result of poor stimulus control or lack of attention to stimuli early in the interval.

The strength of this effect was considered surprising for the following reasons. First, each trial was segmented into 10 different key colors, with one stimulus presented in a fixed temporal position and the remaining nine stimuli randomly resequenced for each trial. In order to come in contact with the unique aspects of the trace stimulus, the bird had to repeatedly attend to all 10 elements of virtually random sequences. Second, a different trace stimulus was used for each different trace interval. The hue that was presented in a fixed position and unpaired with food in a given phase had been randomly presented and paired with food in the prior phase. Finally, there was no 

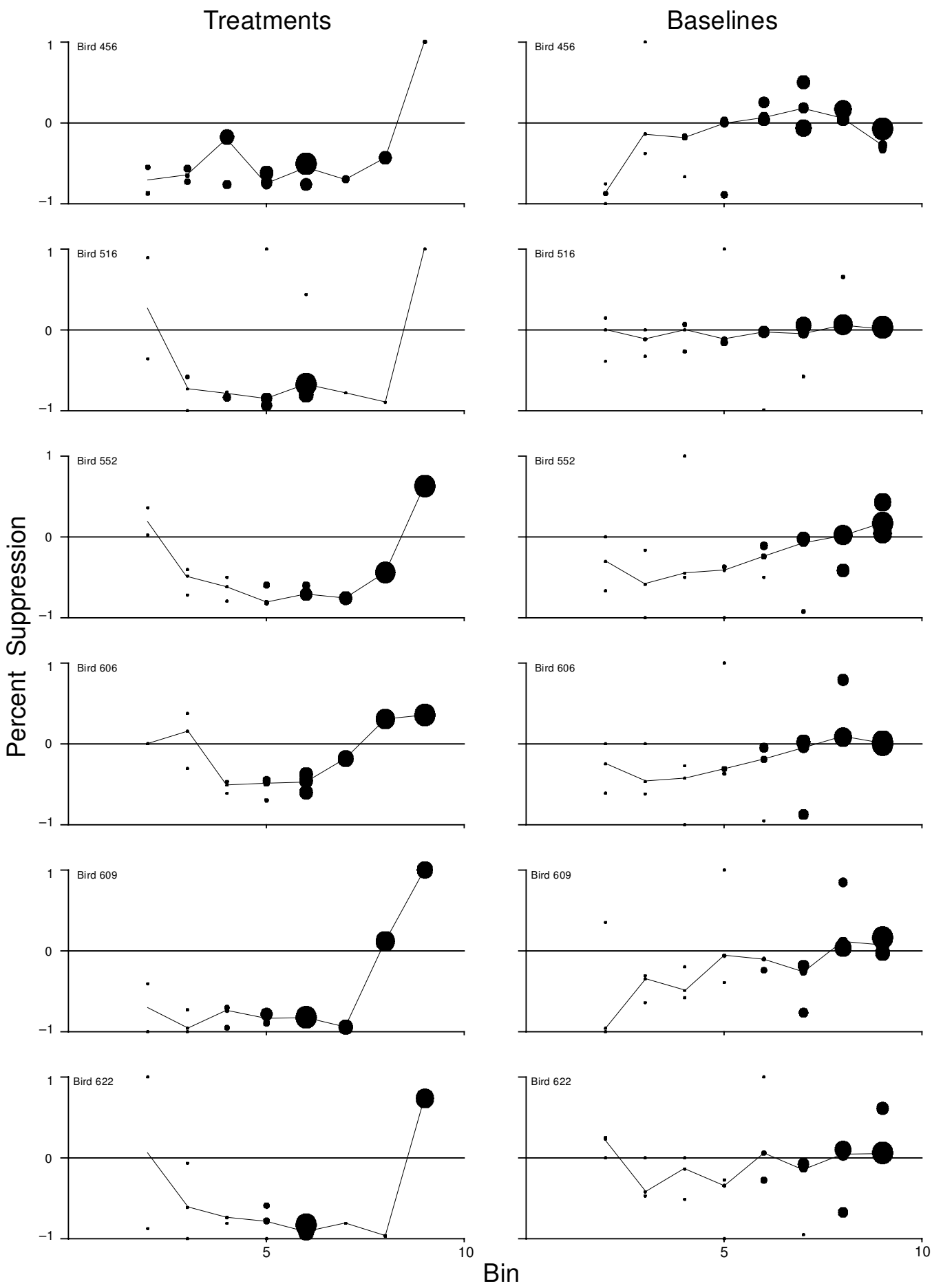

Figure 5. The relative rate change and mean response rate in the adjacent bins for each bird under the treatment (left column) and baseline (right column) conditions. The relative deviation from a spline is depicted as the deviation of a data point from the zero axis, and the response rate in the adjacent bins is represented by the diameter of the data point. The functions through the data points indicate the median percent change in the response rate for each bin.

explicit change in reinforcement rate contingent on a behavior change to the trace stimulus.

The fact that a reliable behavior change occurred to the trace stimuli does not indicate the nature of that con- trol. There are two potential explanations for the obtained behavior change to the trace stimuli in the present study. The first is a modified version of the traditional explanation for trace conditioning. This modified posi- 
tion begins with the role of the relative contingency of the stimulus with the final reinforcer and extends that notion to include a gradient extending from a strong positive correlation with food presentation at the end of the interval to a strong negative correlation with food presentation at the beginning of the interval. This view would suggest that the dominant effect obtained in the present study was the acquired inhibitory control over the terminal behavior of a stimulus correlated with a nonreinforced position in the interfood interval, against an excitatory context provided by the randomized clock and temporal stimuli (Barnet \& Miller, 1996; Dinsmoor et al., 1986; Palya, 1993; Silva \& Timberlake, 1998, 1999).

The alternate position would diminish the role of temporal position and argue that the nine randomly presented stimuli in each phase were each occasionally paired with food presentation and, as a result, were each conditioned; on the other hand, the trace stimulus in each of those procedures was never paired with food, and any conditioning to it would, therefore, be expected to extinguish. From the latter view, the effect obtained in the present study would be the result of an extinguished stimulus in a set of nine distinct excitatory stimuli.

The results from Phase 22 support the latter interpretation, in that an explicitly unpaired stimulus came to control a lower response rate. However, it would appear that this view- that is, that of the neutral trace stimulus compared against a set of nine excitatory stimuli-not entirely appropriate. The results from Phase 23 showed that a trace stimulus could control a rate suppression even when it was also paired with food presentation as often as the other stimuli were. The resolution of the degree to which each of these factors controls the behavior in very long trace procedures awaits further research.

In addition to the rate suppression to the trace stimulus, the present procedures resulted in the characteristically lower rates that occur to portions of the interfood interval that were further removed from the reinforcer. This "temporal effect" could not be attributed to a lack of attention or a failure of control by key stimuli, given that the general rate loss across the interfood interval occurred in birds that exhibited reliable rate changes to very early trace stimuli. The existence of the trace effect indicated that the temporal effect was not due to a lack of stimulus control or a lack of attention, because a specific stimulus out of a set of 10 continued to control differential behavior even when it was in the early portion of the interfood interval. The occurrence of a behavior change to the trace stimulus throughout the interfood interval indicated that behavioral regularities across a fixed temporal interval are more complex than either the inability of any but the very last portion of an interfood interval to condition behavior, or a simple loss of attention. If either of the latter were the case, then the trace stimulus would have failed to maintain differential behavior in the early portions of the interval.

There are several incompatible views of how behavior should equilibriate across the trial to within-trial explicit stimuli other than the stimulus with the greatest contiguity with the upcoming food presentation. For example, information views (see, e.g., Egger \& Miller, 1962; Sutton \& Barto, 1990) argue that the first of a series of stimuli should control the highest rates; a simple discrimination view (see, e.g., Ferster \& Skinner, 1957) argues that only the last stimulus should control behavior; and conditioned reinforcement or higher order conditioning could be invoked, after the fact, to account for virtually any behavior. This discrepancy in prediction and lack of systematic rules of application indicates that the rules for predicting behavior to serial stimuli are not yet sufficiently formal.

Unfortunately, the problem has not received as much research interest as have programs examining behavioral adaptation with increasing experience (Hull, 1943; Rescorla \& Wagner, 1972), behavioral adaptation across an unsignaled temporal interval (Gallistel \& Gibbon, 2000; Miller \& Schachtman, 1985; Spence, 1956), or behavior to concurrently available alternatives (Baum, 1974; Herrnstein, 1970; Mazur, 2002). However, comprehensive principled models that can simultaneously accommodate acquisition, timing, choice, and within-trial events are essential if behavior is to be predicted in a wide variety of situations.

Palya (1993) noted that behavior change with experience and behavior change across a trial were independent forms of dynamic behavior and are most productively depicted as such. He proposed a general conceptual framework that provided a structure within which both acquisition and within-trial measures could be considered. His model specifies the behavior expected to stimuli that have systematically differing correlations with reinforcement, and it provides a continuous surface that depicts the expected behavior at each point in the gradient as a function of each amount of experience. The simplest, best known instantiation of this type of gradient is a fixed interfood interval. However, other procedures that result in an analogous gradient of correlations with the reinforcer produce similar results (Palya \& Bevins, 1990). In a fixed interfood interval, the temporal stimuli at the end of the interval have the highest positive correlation with the reinforcer, whereas stimuli at the onset of the interval have the highest negative correlation with the reinforcer.

A graphical representation of the expected idealized output of Palya's (1993) bipolar model as applied to fixed interfood intervals is provided in Figure 6. The passage of time within a trial is depicted across the $x$-axis, increasing experience is depicted from the front to the back of the figure, and the dependent measure is indicated on the $y$-axis. As can be seen, different behaviors are expected to be controlled by the stimuli most positively and most negatively correlated with the primary reinforcer. The right rear corner of the figure (the end of the trial after much experience) specifies that strong approach or terminal behavior would be expected. The left rear corner (the beginning of the trial after much 


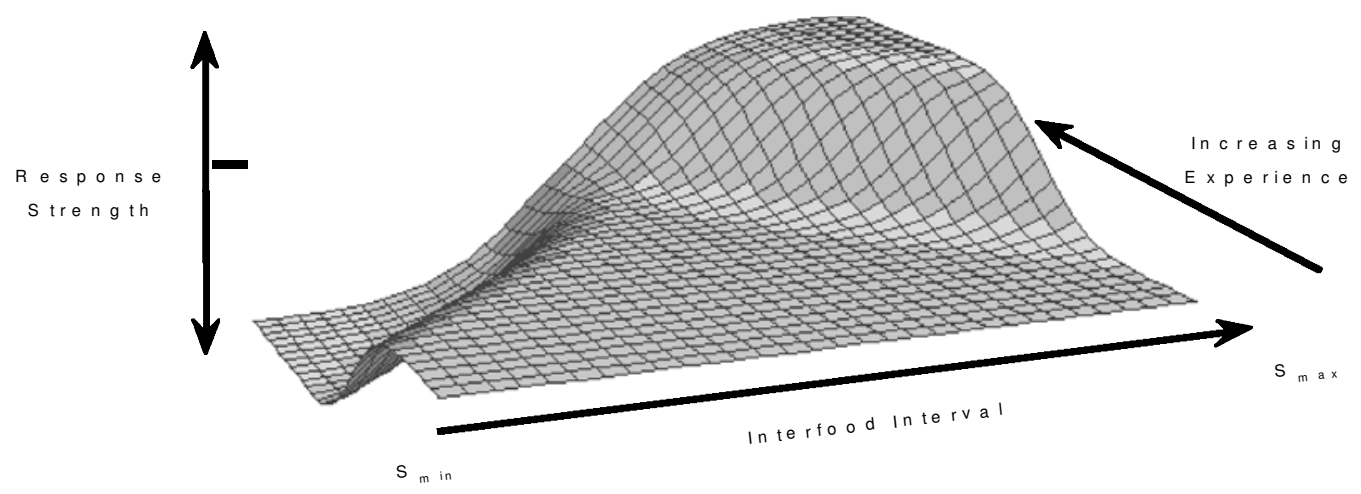

Figure 6. Depiction of the idealized behavioral output predicted by Palya's (1993) bipolar model. Position in the interfood interval is indicated from left to right across the $x$-axis, increasing experience is depicted from front to back, and the strength and vector of the obtained behavior is depicted on the vertical axis.

experience) specifies that strong avoidance or inhibition of the terminal behavior would be expected.

Palya's (1993) bipolar model was based on the findings that stimuli correlated with successively later portions of the second half of an interfood interval functioned as successively stronger reinforcers for the response class that produced them; stimuli correlated with successively earlier portions of the first half of an interfood interval were successively better at supporting their own removal (Dinsmoor et al., 1986; Palya, 1993). Palya (1993) reasoned that both Pavlovian and operant procedures could, therefore, be expected to control behavior in a similar fashion, with opposing vectors occurring at opposite ends of a gradient of correlations with the upcoming reinforcer. Stimuli correlated with the maximum likelihood of the reinforcer $\left(S_{\max }\right)$ would control terminal behavior, whereas stimuli correlated with the minimum likelihood of the reinforcer $\left(\mathrm{S}_{\min }\right)$ would control some other behavior, such as escape, inhibition, or an element from a different behavior system (Dinsmoor, 1983; Palya, 1993; Palya \& Bevins, 1990; Silva \& Timberlake, 1998, 1999). From this perspective, it is possible for all portions of the interval to condition behavior. For example, a bipolar view would argue that the behavior to a clock was indicative of the behavior controlled by each portion of an interval rather than the result of higher order conditioning that never extinguishes. A unipolar view, on the other hand, would argue that conditioning does not occur once the separation from reinforcement exceeds some relatively short duration.

The bipolar model is consistent with any view that argues that stimuli correlated with the reinforcer should control terminal behavior, whereas stimuli signaling the absence of the reinforcer should be inhibitory or control some other behavior. These plausible notions are well supported in the literature (Barnet et al., 1997; Dinsmoor, 1983; Palya, 1993; Silva \& Timberlake, 1998). The output function of the bipolar model could result from a variety of proposed underlying processes (Gallistel \& Gib- bon, 2000; Miller \& Schachtman, 1985; Rescorla \& Wagner, 1972).

The present results provide some support for the bipolar model in that the trace stimuli could acquire control over a behavior change in some birds even in the earliest portions of the interval. Furthermore, control changed from excitatory in the final portions of the interval to inhibitory (with respect to the terminal behavior) in the earlier portions of the interval. Both of these findings are inconsistent with any unipolar view that would suggest that the effects of reinforcement dissipate to zero relatively quickly as a stimulus is further removed from the reinforcer. The present findings also contrast with views suggesting that the early portion of a fixed interfood interval controls little attention to explicit temporally correlated stimuli (Shull, 1979).

However, some details of the present findings were not predicted by Palya's (1993) bipolar model. As is summarized in Figure 5, the crossover between excitatory and inhibitory control did not occur at the approximate midpoint of the interval; the relative suppression or facilitation controlled by the trace stimuli across the interval at equilibrium was relatively dichotomousin form rather than ogival in all but one bird; and the magnitude of the suppression to the trace stimulus did not continue to increase across the earliest portions of the interfood interval. Whether these discrepancies require a substantive modification of the bipolar model or reflect only changes in parameter values or the inappropriate translation of the behavior into numerical indices remains an empirical question.

\section{REFERENCES}

Barnet, R. C., Cole, R. P., \& Miller, R. R. (1997). Temporal integration in second-order conditioning and sensory preconditioning. Animal Learning \& Behavior, 25, 221-233.

BARNET, R. C., \& Miller, R. R. (1996). Temporal encoding as a determinant of inhibitory control. Learning \& Motivation, 27, 73-91.

BAUM, W. M. (1974). On two types of deviation from the matching law: Bias and undermatching. Journal of the Experimental Analysis of Behavior, 22, 231-242. 
Catania, A. C. (1998). Learning (4th ed.). Upper Saddle River, NJ: Prentice-Hall.

DEws, P. B. (1962). The effect of multiple $\mathrm{S}^{\Delta}$ periods on responding on a fixed-interval schedule. Journal of the Experimental Analysis of Behavior, 6, 369-374.

Dinsmoor, J. A. (1983). Observing and conditioned reinforcement. Behavioral \& Brain Sciences, 6, 693-728.

Dinsmoor, J. A., Dougan, J. D., Pfister, J., \& Thiels, E. (1992). The auto-shaping procedure as a residual block clock. Journal of the Experimental Analysis of Behavior, 58, 265-276.

Dinsmoor, J. A., LeE, D. M., \& Brown, M. M. (1986). Escape from serial stimuli leading to food. Journal of the Experimental Analysis of Behavior, 46, 259-279.

DOMJAN, M. (2003). The principles of learning and behavior (5th ed.). Belmont, CA: Wadsworth/Thomson Learning.

EGger, D. M., \& MiLler, N. E. (1962). Secondary reinforcement in rats as a function of information value and reliability of the stimulus. Journal of Experimental Psychology, 64, 97-104.

FARMER, J., \& SCHOENFELD, W. N. (1966). Varying temporal placement of an added stimulus in a fixed-interval schedule. Journal of the Experimental Analysis of Behavior, 9, 369-375.

Ferster, C. B., \& Skinner, B. F. (1957). Schedules of reinforcement. Englewood Cliffs, NJ: Prentice-Hall.

Gallistel, C. R., \& GibBon, J. (2000). Time, rate, and conditioning. Psychological Review, 107, 289-344.

Gibbon, J., \& Balsam, P. (1981). Spreading association in time. In C. M. Locurto, H. S. Terrace, \& J. Gibbon (Eds.), Autoshaping and conditioning theory (pp. 219-253). New York: Academic Press.

Herrnstein, R. J. (1970). On the law of effect. Journal of the Experimental Analysis of Behavior, 13, 243-266.

Hull, C. L. (1943). Principles of behavior. New York: Appleton-CenturyCrofts.

Hull, C. L. (1952). A behavior system. New Haven: Yale University Press.

KILlEeN, P. R. (1994). Mathematical principles of reinforcement. Behavioral \& Brain Sciences, 17, 105-172.

Lucas, G. A., Deich, J. D., \& Wasserman, E. A (1981). Trace autoshaping: Acquisition, maintenance, and path dependence at long trace intervals. Journal of the Experimental Analysis of Behavior, 36, 61-74.

Mazur, J. E. (1998). Learning and behavior (4th ed.). Upper Saddle River, NJ: Prentice-Hall.

MAzUr, J. E. (2002). Evidence against a constant-difference effect in concurrent-chain schedules. Journal of the Experimental Analysis of Behavior, 77, 147-155.

Miller, R. R., \& Schachtman, T. R. (1985). Conditioning context as an associative baseline: Implications for response generation and the nature of conditioned inhibition. In R. R. Miller \& N. E. Spears (Eds.), Information processing in animals: Conditioned inhibition (pp. 51-88). Hillsdale, NJ: Erlbaum.

PALYA, W. L. (1985). Sign-tracking with an interfood clock. Journal of the Experimental Analysis of Behavior, 43, 321-330.

PAlya, W. L. (1993). Bipolar control in fixed interfood intervals. Journal of the Experimental Analysis of Behavior, 60, 345-359.
Palya, W. L., \& Bevins, R. A. (1990). Serial conditioning as a function of stimulus, response, and temporal dependencies. Journal of the Experimental Analysis of Behavior, 53, 65-85.

Palya, W. L., \& Chu, J. Y. M. (1996). Real-time dynamics in the interaction between a trial stimulus and its temporal context. Animal Learning \& Behavior, 24, 92-104.

Palya, W. L., \& Walter, D. E. (1993). A powerful, inexpensive experiment controller or IBM PC interface and experiment control language. Behavior Research Methods, Instruments, \& Computers, 25, 127-136.

Pavlov, I. P. (1927). Conditioned reflexes (G. V. Anrep, Trans.). London: Oxford University Press.

Press, W. H., Flannery, B. P., Teukolsky, S. A., \& Vetterling, W. T. (1989). Numerical recipes: The art of scientific computing (FORTRAN version). New York: Cambridge University Press.

Rescorla, R. A. (1967). Pavlovian conditioning and its proper control procedures. Psychological Review, 74, 71-80.

Rescorla, R. A., \& WAgner, A. R. (1972). A theory of Pavlovian conditioning: Variations in the effectiveness of reinforcement and nonreinforcement. In A. H. Black \& W. F. Prokasy (Eds.), Classical conditioning II: Current research and theory (pp. 64-99). New York: Appleton-Century-Crofts.

Shull, R. L. (1979). The postreinforcement pause: Some implications for the correlational law of effect. In M. D. Zeiler \& P. Harzem (Eds.), Reinforcement and the organization of behaviour (pp. 193-221). New York: Wiley.

Silva, K. M., \& Timberlake, W. (1998). A behavior systems view of responding to probe stimuli during an interfood clock. Animal Learning \& Behavior, 26, 313-325.

Silva, K. M., \& TimberlaKe, W. (1999). Rats' behavior during an interfood clock is altered by the temporal pattern of interfood stimuli. Learning \& Motivation, 30, 183-200.

SPence, K. W. (1956). Behavior theory and conditioning. New Haven: Yale University Press.

Sutton, R. S., \& Barto, A. G. (1990). Time derivative models of Pavlovian reinforcement. In M. R. Gabriel \& J. W. Moore (Eds.), Learning and computational neuroscience: Foundations of adaptive networks (pp. 497-537). Cambridge, MA: MIT Press.

Thorndike, E. L. (1898). Animal intelligence: An experimental study of the associative processes in animals. Psychological Review Monograph Supplements, 2(4), 1-10.

TUFTE, E. R. (2001). The visual display of quantitative information (2nd ed.). Cheshire, CT: Graphics Press.

Wright, A. A. (1978). Construction of equal-hue discriminability scales for the pigeon. Journal of the Experimental Analysis of Behavior, 29, 261-266.

(Manuscript received June 17, 2002; accepted for publication September 12,2002.) 